\title{
Hábitos y factores de riesgo cardiovascular en estudiantes de primer semestre de la Universidad Santo Tomás*
}

\author{
Henry Humberto León Ariza*, Aura Catalina Zea Robles ${ }^{* * *}$
}

\begin{abstract}
Recibido: 24 de septiembre de 2009 Revisado: 11 de diciembre de 2009 Aprobado: 6 de febrero de 2010
\end{abstract}

\section{Resumen}

Las enfermedades cardiovasculares son la primera causa de muerte en Colombia y en el mundo; se sabe que su origen es multifactorial. Se realizó un estudio descriptivo en el que participaron 289 estudiantes de pregrado de la Universidad Santo Tomas de Bogotá. Los participantes respondieron una encuesta de hábitos y antecedentes médicos y familiares, y fueron evaluados tanto antropométricamente como en su presión arterial. De los 289, 80,3\% tenían al menos un antecedente familiar de importancia para riesgo cardiovascular. 55\% fueron hombres y $45 \%$ mujeres. El rango de edad para el grupo masculino fue de 16,5 y 21,7 años, y para el femenino fue de 19 y 24 años. El estudio mostró una alta prevalencia de sedentarismo, antecedentes patológicos familiares y tabaquismo asociados con sobrepeso y cifras prehipertensivas en una población de estudiantes muy jóvenes, cuya apariencia general pudiera catalogarse como saludable y en quienes ningún médico o profesional de las ciencias del deporte anticiparía algún riesgo durante la actividad deportiva. Surge la necesidad de establecer programas que promuevan hábitos saludables de vida en los estudiantes jóvenes y prevenir la posible presencia de enfermedades cardiovasculares en la edad adulta.

\section{Palabras clave}

Enfermedades cardiovasculares, sobrepeso y obesidad, sedentarismo, tabaquismo, hipertensión, enfermedades crónicas no transmisibles

* Artículo de investigación, realizado por el área de salud de la cátedra Formación Fissica Integral Henri Didón, Departamento de Humanidades y Formación Integral, Universidad Santo Tomás, sede Bogotá.

** Licenciado en Educación Física: Universidad Pedagógica Nacional; Médico Cinjano: Universidad Nacional de Colombia, Coordinador del área de la salud: Cátedra Formación Física Integral Henri Didón, docente director de la linea de investigación en fisiología muscular. Facultad de Cultura Fisica, Deporte y Recreación, Bogotá. preparacionfisicą@ gmail.com.

*** Profesional en Ciencias del Deporte: Universidad de Ciencias Aplicadas y Ambientales, UDCA, Fisioterapeuta en formación: Universidad Nacional de Colombia, Docente cátedra Formación Física Integral Henri Didón, Bogotá. aucazero@ hotmail.com. 


\title{
Habits and risk factors for cardiovascular diseases on first semester students at Universidad Santo Tomás
}

\author{
Henry Humberto León Ariza, Aura Catalina Zea Robles
}

\begin{abstract}
Cardiovascular disease (CVD) is the first cause of death in Colombia and is the most common cause of death worldwide. It is known that CVD originates from multiple factors. A descriptive study was conducted on 289 undergraduate students at University of Saint Thomas, in Bogota. All participants were asked to answer a survey on the habits and medical and family background. In addition, anthropometric examination and blood pressure measurements were carried out on all of them. Out of the 289 participants $80.3 \%$ was found to have at least one major familial risk factor for CVD. 55\% of the population was constituted by males (age range16.5 to 21.7 years) and $45 \%$ by females (age range 19 to 24 years). This study has shown that high prevalence of sedentary life-style, pathologies on the family background, and smoking habits were all associated with overweigh and pre-hypertensive values among very young students which apparent health status at first glance might be reported as healthy and without any risk important to be assessed previous to physical exercise activities participation. Establishing programs to promoting healthy life-style habits among young students is urgently needed in order to prevent the development of CVD.
\end{abstract}

\section{Key words}

Cardiovascular disease, overweight and obesity, sedentarism, smoking, hypertension, chronic diseases non communicable. 


\section{INTRODUCCIÓN}

Las enfermedades que afectan el sistema cardiovascular (ECV) son en la actualidad la primera causa de muerte en adultos a nivel mundial (Lloyd, D., et al., 2009); ya desde hace cerca de 50 años uno de los estudios epidemiológicos más grandes a nivel mundial, denominado el estudio de Framingham (Dawber, T.R., 1980), demostró que el origen de las ECV es multifactorial $y$ que se encuentra fuertemente asociado a otras enfermedades, como la obesidad (Daniels, S.R., et al., 2005), la ateroesclerosis temprana (Berenson, G.S., et al., 1998), los valores altos de colesterol (Assmann, G., 2006), el tabaquismo (Craig, W.Y., 1989) y especialmente el sedentarismo (Darren, E.R., 2006), haciendo que los esfuerzos para evitar la muerte prematura por ECV se centren en el control de estos factores de riesgo de la forma más oportuna posible.

Siguiendo la tendencia mundial, en Colombia la primera causa de muerte en mayores de 45 años, hombres y mujeres, también lo es la enfermedad cardiovascular (Ministerio de Protección Social, 2008). Estudios realizados anteriormente muestran que en nuestro país la prevalencia durante la etapa escolar (15 a 18 años) de consumo de alcohol y tabaquismo es cercana al 63,5\% y al $13,9 \%$, respectivamente (Uscátegui, et al., 2003), y que estos valores se incrementan con el paso a la etapa universitaria (Hernández, J., et al., 2006, Dirección Nacional de Estupefacientes, 2001); resultados similares se han observado en otros comportamientos nocivos como el incremento de la dieta aterogénica, el estrés y el sedentarismo (Sánchez, M., et al., 2009).

Este cambio comportamental al momento de ingresar a la universidad se asocia con una cultura que falsamente muestra a los jóvenes libres de hacer lo que les plazca, con nuevos criterios de aceptación social y libertades económicas (Londoño, C., et al., 2005); todo esto lastimosamente crea un ambiente propicio para el desarrollo de los ya nombrados factores de riesgo cardiovascular.

Dado lo anterior encontramos de sumo interés investigar algunos factores de riesgo de nuestra población universitaria, y para ello analizamos los estudiantes de primer semestre, porque consideramos que el paso de la educación media vocacional a la superior es un momento clave para la intervención en hábitos saludables de vida.

\section{Metodología}

El siguiente es un estudio descriptivo correlacional, la población se constituyó con estudiantes de primer semestre de todas las carreras ofrecidas por la Universidad Santo Tomas en la sede Bogotá, durante el segundo semestre de 2009, ambos géneros, con el objetivo de establecer comparaciones estadísticamente significativas entre las variables. Fijando un error máximo en el $5 \%$, con una prevalencia del $50 \%$, para un intervalo de confianza del 95\%, la muestra mínima resultó ser de 269 individuos, con sobremuestreo hasta 306 para incrementar la representatividad; se retiraron del estudio 17 individuos porque entregaron datos incompletos, por lo cual la muestra final fue de 289 estudiantes.

Para la selección de la muestra se escogieron al azar doce grupos de la cátedra Formación Física Integral Henri Didón, materia ofrecida los días lunes, miércoles, viernes y sábados a todos los estudiantes de primer semestre de la universidad. 
Las variables analizadas fueron: género, edad, facultad (Ingeniería Ambiental, Economía, Comunicación Social, Ingeniería Civil, Negocios Internacionales, Contaduría Pública, Cultura Física, Deporte y Recreación, Ingeniería Electrónica, Psicología, Licenciatura en Filosofía y Lengua Castellana, Derecho, Ingeniería de Telecomunicaciones, Administración de Empresas, Sociología, Ingeniería Mecánica y Estadística), hábito de fumar, actividad física, índice de masa corporal, porcentaje de tejido adiposo, presión arterial en reposo, perímetro abdominal y antecedentes familiares (obesidad, diabetes, hipertensión, hipercolesterolemia e infarto agudo del miocardio).

El IMC se calculó como el cociente entre peso/talla ${ }^{2}\left(\mathrm{~kg} / \mathrm{m}^{2}\right)$ (OMS, 2009), y para su determinación se usaron valores fijados objetivamente (balanza calibrada y tallímetro estandarizado de pared).

La presión arterial se midió con el participante sentado, en el brazo derecho, sin actividad física previa y con un descanso mínimo de 15 minutos.

El porcentaje de tejido adiposo se calculó mediante la fórmula de Yuhasz (1974) para hombres y mujeres entre 18 a 30 años; la toma de los pliegues se hizo siguiendo el protocolo estandarizado (Lohman, T.G., et al., 1991), se utilizó un calibrador de pliegues marca Holtain, con una presión constante de $10 \mathrm{~g} / \mathrm{mm}^{2}$ de superficie de contacto y precisión $0,2 \mathrm{~mm}$; durante todo el estudio un solo evaluador tomó todas las mediciones; los valores obtenidos se compararon con las tablas que sugirió Hoeger (1986) para hombres y mujeres menores de 20 años.

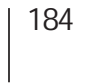

El perímetro abdominal setomó a la altura del ombligo y la comparación de los valores se hizo de acuerdo con las tablas establecidas para Colombia (Pérez, M., et al., 2003).

\section{Análisis estadístico}

Los datos recolectados se almacenaron en una base de datos en el programa Excel 2007 (Microsoft Corporation), la cual se analizó mediante el programa estadístico SPSS versión 17 (SPSS Inc.). Para describir las variables se emplearon medidas de tendencia central (promedios) y de dispersión (desviación estándar, DS); para probar la asociación de variables se utilizó prueba $t$, con un nivel de significancia de 0,05 y 0,005.

Desde el punto de vista ético los métodos empleados son inocuos, por lo que se clasifica como una investigación con riesgo mínimo, según la Resolución 8430 de 1993 del Ministerio de Salud de la República de Colombia. Todo lo consignado fue estrictamente privado y confidencial.

\section{Definiciones utilizadas en el análisis de datos}

Fumador: Persona que fuma en la actualidad, independientemente de la cantidad consumida.

Sedentario: Persona que durante sus actividades de ocio diarias consume el equivalente a 4 o menos $\mathrm{MET}^{11}$ y estas no superan los 30 minutos (Cabrera, A., y cols. 2007).

Antecedentes familiares: Antecedentes de diabetes mellitus, hipertensión arterial,

\footnotetext{
11 Una MET (tasa de intercambio metabólico) es una unidad que se utiliza para cuantificar las actividades que comúnmente se realizan: entre más enérgica sea la actividad, le corresponde un número mayor. Su cuantificación se hace con encuestas diseñadas para tal fin (Bouchard, C. y cols. 1983).
} 
obesidad, valores altos de colesterol (hipercolesterolemia) y enfermedades cardiovasculares, padecidos por familiares de hasta segundo grado de consanguinidad.

\section{Resultados}

En el estudio participaron un total de 289 estudiantes cuyas características se describen en la tabla 1. El 55\% de los participantes fueron del género masculino y $45 \%$, del género femenino; la edad promedio fue de 18,9 años, ubicándose el 55\% de la población entre los 16 y 18 años; la facultad donde se analizaron más estudiantes $(13,1 \%)$ fue Ingeniería Ambiental, mientras que de Ingeniería Mecánica y Estadística solo el $0,7 \%$ en ambos casos.

Tabla 1. Características de la muestra de estudiantes de primer semestre de la Universidad Santo Tomas durante el segundo semestre de 2009

\begin{tabular}{|l|c|c|}
\hline Variable & $\mathrm{n}$ & $\%$ \\
\hline Género & 159 & $55,0 \%$ \\
\hline Masculino & 130 & $45,0 \%$ \\
\hline Femenino & 289 & $100,0 \%$ \\
\hline Total & \multicolumn{3}{|l|}{} \\
\hline Edad (años) & 159 & $55,0 \%$ \\
\hline $16-18$ & 94 & $32,5 \%$ \\
\hline $19-21$ & 24 & $8,3 \%$ \\
\hline $22-24$ & 12 & $4,2 \%$ \\
\hline$>25$ & 289 & $100,0 \%$ \\
\hline Total & \multicolumn{2}{|l}{} \\
\hline
\end{tabular}

\begin{tabular}{|c|c|c|}
\hline \multicolumn{3}{|l|}{ Facultad } \\
\hline Ingeniería Ambiental & 38 & $13,1 \%$ \\
\hline Economía & 37 & $12,8 \%$ \\
\hline Comunicación Social & 30 & $10,4 \%$ \\
\hline Ingeniería Civil & 27 & $9,3 \%$ \\
\hline Negocios Internacionales & 25 & $8,7 \%$ \\
\hline Contaduría Pública & 25 & $8,7 \%$ \\
\hline $\begin{array}{l}\text { Cultura física, Deporte y } \\
\text { Recreación }\end{array}$ & 20 & $6,9 \%$ \\
\hline Ingeniería Electrónica & 19 & $6,6 \%$ \\
\hline Psicología & 18 & $6,2 \%$ \\
\hline $\begin{array}{l}\text { Licenciatura en Filosofía y Lengua } \\
\text { Castellana }\end{array}$ & 15 & $5,2 \%$ \\
\hline Derecho & 14 & $4,8 \%$ \\
\hline Ingeniería de Telecomunicaciones & 7 & $2,4 \%$ \\
\hline Administración de Empresas & 6 & $2,1 \%$ \\
\hline Sociología & 4 & $1,4 \%$ \\
\hline Ingeniería Mecánica & 2 & $0,7 \%$ \\
\hline Estadística & 2 & $0,7 \%$ \\
\hline Total & 289 & $100,0 \%$ \\
\hline
\end{tabular}

El promedio de índice de masa corporal (IMC) en los hombres fue de $21,4 \mathrm{~kg} / \mathrm{m}^{2}$ DS $\pm 3,5$, y en las mujeres fue $21,7 \mathrm{~kg} / \mathrm{m}^{2} \mathrm{DS} \pm$ 3,0; el perímetro abdominal promedio para los hombres fue de $78,7 \pm 9,3 \mathrm{~cm}$ y en las mujeres, $78,2 \pm 9,0$. El porcentaje de grasa fue de $13,4 \% \pm 4,7$ y de $29,2 \% \pm 5,9$ en hombres y mujeres, respectivamente. Por último, la presión arterial sistólica (PAS) y la presión arterial diastólica (PAD) fue 115,1/68,9 y $105,5 / 64,4 \mathrm{~mm} / \mathrm{Hg}$, en hombres y mujeres (ver tabla 2). 
Hábitos y factores de riesgo cardiovascular en estudiantes de primer semestre de la Universidad Santo Tomás

Tabla 2. Características antropométricas generales de los estudiantes de primer semestre discriminados por género

\begin{tabular}{|l|c|c|c|}
\hline & Hombres & Mujeres & $p$ \\
\hline IMC (DS) & $21,4(3,5)$ & $21,7(3,0)$ & NS \\
\hline Perímetro abdominal (DS) & $78,7(9,3)$ & $78,2(9,0)$ & NS \\
\hline$\%$ Grasa (DS) & $13,4(4,7)$ & $29,2(5,9)$ & $<0,005$ \\
\hline Presión arterial sistólica en $\mathrm{mm} / \mathrm{Hg}$ (DS) & $115,1(11,2)$ & $105,5(10,4)$ & $<0,005$ \\
\hline Presión arterial diastólica en $\mathrm{mm} / \mathrm{Hg}(\mathrm{DS})$ & $68,9(7,9)$ & $64,4(6,7)$ & $<0,005$ \\
\hline (DS) Desviación estándar, NS No significativo & & \\
\hline
\end{tabular}

El $13,8 \%$ de los hombres y el 16,2\% de las mujeres presentaron un IMC superior a 25 $\mathrm{kg} / \mathrm{m}^{2}$ (sobrepeso) y el 1,9\% de los hombres y el $0,8 \%$ de las mujeres tienen un IMC superior a $30 \mathrm{~kg} / \mathrm{m}^{2}$ (obesidad). 5,7\% de los hombres tienen un perímetro abdominal superior a $94 \mathrm{~cm}$, y un 7,7\% de las mujeres un perímetro abdominal superior a $80 \mathrm{~cm}$ (un perímetro abdominal superior a $94 \mathrm{~cm}$ en hombres y $80 \mathrm{~cm}$ en mujeres se asocia a riesgo cardiovascular alto). Cuando se comparó el porcentaje de tejido adiposo con las tablas sugeridas por Hoeger, se encontró que el 62,3\% de los hombres tenían un porcentaje de tejido adiposo óptimo para la edad, un 34,6\% tenían porcentaje de grasa calificado como bueno, un $16,2 \%$ tenían un porcentaje moderado, un $7,7 \%$ uno alto $\mathrm{y}$ un $1,5 \%$ muy alto. En el caso de las mujeres, un $1,5 \%$ tenían un porcentaje óptimo, $10,8 \%$ bueno, $25,4 \%$ moderado, $33,1 \%$ alto y $29,2 \%$ muy alto (ver tabla 3 ).
Tabla 3. Índice de masa corporal, perímetro abdominal y porcentaje de grasa en hombres y mujeres participantes del estudio

\begin{tabular}{|l|r|r|}
\hline \multicolumn{1}{|c|}{ IMC $^{*}$} & Hombres & Mujeres \\
\hline$<18,5(\%)$ & $29(18,2)$ & $16(12,3)$ \\
\hline $18,5-24,9(\%)$ & $108(67,9)$ & $93(71,5)$ \\
\hline $25-29,9(\%)$ & $19(11,9)$ & $20(15,4)$ \\
\hline $30-34,9(\%)$ & $2(1,3)$ & $1(0,8)$ \\
\hline $35-39.9(\%)$ & $1(0,6)$ & $0(0,0)$ \\
\hline Perímetro abdominal \\
\hline$>94$ cm (\%) & $9(5,7)$ & NA \\
\hline$>102$ cm (\%) & $6(3,8)$ & NA \\
\hline$>80$ cm (\%) & NA & $10(7,7)$ \\
\hline$>88$ cm (\%) & NA & $1(0,8)$ \\
\hline$\%$ Grasa & $81(62,3)$ & $2(1,5)$ \\
\hline Óptimo (\%) & $45(34,6)$ & $14(10,8)$ \\
\hline Bueno (\%) & $21(16,2)$ & $33(25,4)$ \\
\hline Moderado (\%) & $10(7,7)$ & $43(33,1)$ \\
\hline Alto (\%) & $2(1,5)$ & $38(29,2)$ \\
\hline Muy alto (\%) & Índice de masa corporal expresado en kg/m² \\
NA: No aplica & & \\
\hline
\end{tabular}

Los resultados de la presión arterial comparados con el séptimo informe Joint National Comitte para la prevención, detección, evaluación y tratamiento de la hipertensión ar- 
terial (Chobanian, A.V., et al., 2003) mostraron que el $27 \%$ de los hombres tenían cifras prehipertensivas comparado con un 5,4\% de las mujeres, y que el 3,1\% de los hombres y el $0,8 \%$ de las mujeres tenían valores para ser considerados hipertensos (ver tabla 4).

Tabla 4. Datos de presión arterial sistólica y diastólica en hombres y mujeres, clasificación según el JNC VII

\begin{tabular}{|l|c|c|}
\hline Presión arterial sistólica & Hombres & Mujeres \\
\hline$<120 \mathrm{~mm} / \mathrm{Hg}$ & $71,1 \%$ & $93,8 \%$ \\
\hline $120-140 \mathrm{~mm} / \mathrm{Hg}$ & $27,0 \%$ & $5,4 \%$ \\
\hline$>140 \mathrm{~mm} / \mathrm{Hg}$ & $1,9 \%$ & $0,8 \%$ \\
\hline Presión arterial diastólica & Hombres & Mujeres \\
\hline$<80 \mathrm{~mm} / \mathrm{Hg}$ & $93,7 \%$ & $99,2 \%$ \\
\hline $80-90 \mathrm{~mm} / \mathrm{Hg}$ & $3,1 \%$ & $0,0 \%$ \\
\hline$>90 \mathrm{~mm} / \mathrm{Hg}$ & $3,1 \%$ & $0,8 \%$ \\
\hline
\end{tabular}

Al organizar a los estudiantes por grupos etarios se encontró que el sedentarismo en los hombres es más prevalente en el grupo de edad de 16 a 18 años, mientras que en las mujeres fue en el grupo de 19 a 21 años; por otro lado, el hábito de fumar es más prevalente en el grupo de hombres de 19 a 21 años, mientras en las mujeres lo fue en el de 22 a 24 años (ver tabla 5).

Tabla 5. Distribución de sedentarismo y tabaquismo por grupos de edad

\begin{tabular}{|l|l|l|l|l|l|l|}
\hline Edad & \multicolumn{5}{|l|}{ Sedentarismo } & \multicolumn{2}{l|}{ Tabaquismo } \\
\hline & Hombres & Mujeres & $p$ & Hombres & Mujeres & $p$ \\
\hline $16-18$ & $66,7 \%$ & $82,1 \%$ & $<0,05$ & $66,7 \%$ & $82,1 \%$ & $<0,005$ \\
\hline $19-21$ & $53,7 \%$ & $87,5 \%$ & $<0,005$ & $53,7 \%$ & $87,5 \%$ & $<0,005$ \\
\hline $22-24$ & $38,9 \%$ & $83,3 \%$ & $<0,05$ & $38,9 \%$ & $83,3 \%$ & NS \\
\hline$>25$ & $50,0 \%$ & $66,7 \%$ & NS & $50,0 \%$ & $66,7 \%$ & NS \\
\hline Todos & $58,5 \%$ & $83,1 \%$ & $<0,005$ & $58,5 \%$ & $83,1 \%$ & $<0,005$ \\
\hline NS: No significativo & \multicolumn{7}{|l}{} \\
\hline
\end{tabular}

El $80,3 \%$ de los estudiantes participantes declararon uno o más antecedentes familiares: obesidad, diabetes, hipertensión, hipercolesterolemia y/o infarto agudo del miocardio. Considerando los mismos de forma independiente: obesidad 29,1\%, diabetes $41,5 \%$, hipertensión 49,1\%, hipercolesterolemia $36,3 \%$ e infarto agudo del miocardio $29,4 \%$.

\section{Conclusión}

Los resultados de esta investigación son similares a los encontrados en otros estudios universitarios y al igual que estos ofrecen conclusiones preocupantes que destacan la importancia de la detección y manejo oportuno de los factores de riesgo cardiovascular en jóvenes, a pesar de las limitaciones de nuestro estudio donde no se tuvieron en 
cuenta los valores de colesterol, que se consideran un factor de riesgo fundamental.

Un estudio similar realizado en Chile (Chiang, M.T., et al., 1991) mostró una prevalencia de tabaquismo de $46,1 \%$, sedentarismo de $47,8 \%$ en hombres y $73,7 \%$ en mujeres, obesidad de 1,9\%, e hipertensión arterial de $4,6 \%$, resultados muy similares a los nuestros. De la misma forma, cuando se comparan los resultados de antecedentes familiares con los obtenidos en Argentina (Girotto, C.A., et al., 1996), nuestra población presenta una mayor prevalencia de estos, especialmente de diabetes, obesidad e hipercolesterolemia.

Este estudio muestra que los factores de riesgo modificables más prevalentes fueron el sedentarismo y el tabaquismo. Los resultados muestran que las mujeres son más sedentarias $(83,1 \%)$ que los hombres $(58,5 \%)$, con datos muy altos especialmente en las mujeres, lo cual es preocupante ya que es bien sabido que el sedentarismo predispone al desarrollo de múltiples enfermedades (Darren, E.R., et al., 2006). Conductas como estas son sin lugar a dudas el resultado de hábitos adquiridos en la infancia, probablemente asociados al uso excesivo de la televisión, el computador y los videojuegos (Gregory, J., et al., 2005).

Por otro lado, también se presentan valores altos de consumo de tabaco, siendo más prevalente en hombres $(43,4 \%)$ que en mujeres $(12,9 \%)$. Es interesante tener en cuenta que a pesar de las campañas antitabaco adelantadas por el gobierno nacional y por los diferentes establecimientos de educación superior, los datos de consumo de tabaco son altos y se relacionan fuertemente con el consumo inicial de tabaco desde la época escolar.
Aunque se encontró un porcentaje muy bajo de obesidad (1,9\% en los hombres y $0,8 \%$ en las mujeres), la cantidad de estudiantes con sobrepeso nos hace pensar que existe una tendencia a que el peso aumente en ellos dentro de algunos años; por otro lado, si a los datos de sobrepeso y obesidad les agregamos los valores obtenidos en la adipometría, el resultado podría asociarse a una alta deficiencia de masa muscular en los estudiantes. Múltiples estudios demuestran que el aumento del tejido adiposo y también la deficiencia de calidad muscular se asocian con el desarrollo de enfermedades crónicas no transmisibles (Wolfe, R.R., 2006).

El perímetro abdominal (PA) como indicador de riesgo cardiovascular representa la acumulación de tejido adiposo a nivel de los órganos internos (Katzmarzyk, P.T., et al., 2006); en nuestro estudio el PA era elevado en un pequeño porcentaje de estudiantes (5,7\% de hombres y 7,7\% de mujeres), como se observa, con una tendencia a ser mayor en el género femenino. Ampliamente se ha demostrado que la acumulación de tejido graso a nivel visceral se asocia con alteraciones hormonales secundarias fuertemente relacionadas con el desarrollo de otras enfermedades, como la diabetes, la hipertensión y en últimas enfermedades cardiovasculares (Patrick, M., et al., 2009).

En relación con la hipertensión arterial, se encontró una prevalencia del 3,1\% en hombres y del 0,8\% en mujeres, demostrándose una asociación estadística con el peso corporal tanto en hombres como en mujeres; cabe señalar que los casos detectados como hipertensos se remitieron a evaluación médica para su seguimiento y control. 
Los antecedentes familiares de enfermedades, como obesidad, hipertensión, hipercolesterolemia, diabetes e infarto agudo del miocardio, constituyen uno de los primeros aspectos que se deben tener en cuenta a la hora de evaluar el riesgo cardiovascular de cualquier individuo; en los estudiantes se presentaron claros antecedentes familiares de al menos una de estas enfermedades (algunos estudiantes tenían un conocimiento limitado de sus antecedentes).

Durante el desarrollo de la cátedra Formación Física Integral Henri Didon, todos los estudiantes recibieron al menos una charla sobre los signos, los síntomas y las consecuencias de padecer estas enfermedades, como parte del programa.

Los resultados del presente estudio deberían hacernos reflexionar sobre el impac-

\section{REFERENCIAS BIBLIOGRÁFICAS}

Assmann, G. (2006). Dyslipidaemia and global cardiovascular risk: clinical issues. European Heart Journal Supplements 8 (Supplement F), F40-F46.

Berenson, G.S., Srinivasan, S.R., Bao, W., et al. (1998). Association between multiple cardiovascular risk factors and atherosclerosis in children and young adults. New England Journal of Medicine, (338), 1650-1656.

Bouchard, C., Tremblay, A., Leblanc, C., Lortie, G., Savard, R. \& Thériault, G. (1983). A method to assess energy expenditure in children and adults. American Journal of Clinical Nutrition, (37), 461-467. to que tiene el paso por la universidad en la salud de los estudiantes, y con base en ello encaminarnos al desarrollo de cursos complementarios de actividad física y hábitos saludables de vida abiertos a todas las carreras, durante la permanencia en la universidad, de manera que podamos adelantarnos a la prevención de enfermedades crónicas no transmisibles y formar no solo profesionales capacitados en sus áreas propias del conocimiento, sino también seres humanos saludables, autoconscientes de su propia salud.

Por otro lado, nos genera la inquietud por realizar nuevos estudios en donde se tengan en cuenta, además de los ya valorados, los factores de riesgo lipídicos. Por último, uno de nuestros objetivos es poder valorar más adelante el efecto de nuestra intervención a través de la cátedra en estudiantes de último semestre de la universidad.

Cabrera, A., Rodríguez, M. \& Rodríguez, L.M. (2007). Sedentarismo: tiempo de ocio activo frente a porcentaje del gasto energético. Revista Española de Cardiología, 60, (3), 244-250.

Chiang, M.T., Casanueva, V., Cid, X., González, U., Olate, P., Nickel. F., et al. (1999). Factores de riesgo cardiovascular en estudiantes universitarios chilenos. Salud Pública de México, (41), 444-451.

Chobanian, A.V., Bakris, G.L., Black, H.R., Cushman, W.C., Green, L.A., Izzo, J.L., et al. (2003). The seventh report of the Joint National Committee on Prevention, Detection, Evaluation, and Treat- 
ment of High Blood Pressure: The JNC 7 Report. The Journal of the American Medical Association, JAMA, (289), 25602572.

Craig, W.Y., Palomaki, G.E. \& Haddow, J.E. (1989). Cigarette smoking and serum lipid and lipoprotein concentrations: an analysis of published data. British Medical Journal, BMJ (298), 784-788.

Daniels, S.R., Arnett, D.K., Eckel, R.H., et al. (2005). Overweight in children and adolescents: pathophysiology, consequences, prevention, and treatment. Circulation, (111), 1999-2012.

Darren, E.R., Warburton, C.W. \& Shannon, S.D. (2006). Health benefits of physical activity: the evidence. Canadian Medical Association Journal, CMAJ, 174, (6), 801809.

Dawber, T.R. (1980). The framingham study. Cambridge, Harvard University Press.

Dirección Nacional de Estupefacientes. (2001). Encuesta nacional sobre consumo de sustancias psicoactivas en jóvenes de 10 a 24 años de edad. Bogotá: Presidencia de la República.

Girotto, C.A., Vacchino, M.N., Spillmann, C.A. \&. Soria, J.A. (1996). Prevalencia de factores de riesgo cardiovascular en ingresantes universitarios. Rev. Saúde Pública, 30 (6), 576-586.

Gregory, J., Béatrice, S. \& James, S. (2005). Psychosocial and Environmental Correlates of Adolescent Sedentary Behaviors. Pediatrics, (116), 908-916.

Hernández, J., Guevara, C.L., García, M.F. \& Tascón, J.E. (2006, enero-marzo). Hábito de fumar en los estudiantes de primeros semestres de la Facultad de Salud, Características y percepciones. Colombia Médica (37) No. 1. Universidad del Valle.
Publicación electrónica: http://colombiamedica.univalle.edu.co/Vol37No1/ Cm37n1\%20html/Cm37n1a4.htm

Hoeger, W. (1986). Lifetime physical fitness and wellness. A personalized program. Colorado, USA.

Katzmarzyk, P.T., Janssen, I., Ross, R., et al. (2006). The importance of waist circumference in the definition of metabolic syndrome: prospective analyses of mortality in men. Diabetes Care, 29, (2) 404-409.

Lloyd, D., Adams, R., Carnethon., M., et al. (2009). Heart disease and stroke statistics-2009 update: a report from the American Heart Association Statistics Committee and Stroke Statistics Subcommittee. Circulation, (119), 480-486.

Lohman, T.G., Roche, A.F. \& Martorell, R. (1991). Anthropometric standardization reference manual, Human Kinetics Books.

Londoño, C., García. W., Valencia, S.C. \& Vinaccia, S. (2005, diciembre). Expectativas frente al consumo de alcohol en jóvenes universitarios colombianos. Anales de Psicología, 2, (21), 259-267.

Ministerio de protección social. (2008). Indicadores básicos. Situación de salud en Colombia. www.minproteccionsocial. gov.co.

Organización Mundial de la Salud - Centro de prensa. Obesidad y sobrepeso. http://www.who.int/mediacentre/factsheets/fs311/es/index.html, Consultado noviembre del 2009.

Patrick, M., Paul, P. \& Philippe, P. (2009). Visceral Obesity: The Link Among Inflammation, Hypertension, and Cardiovascular Disease. Hypertension, (53), 577-584. 
Pérez, M., Casas, J.P., Cubillos-Garzón, L.A., Serrano, N.C., Silva, F., Morillo, C.A., et al. (2003). Using waist circumference as a screening tool to identify Colombian subjects at cardiovascular risk. European Journal of Cardiovascular Prevention and Rehabilitation, (10), 328-335.

Sánchez, M., Moreno, G.A., Marín, M.E. \& García, L.H. (2009). Factores de riesgo cardiovascular en poblaciones jóvenes. Rev. Salud Pública, 11, (1), 110-122.
Uscátegui, R.M., Álvarez, M.C. \& Laguado, I., et al. (2003). Factores de riesgo cardiovascular en niños de 6 a 18 años de Medellín (Colombia). Anales de Pediatría $58,(5), 411-417$.

Wolfe, R.R. (2006). The underappreciated role of muscle in health and disease. American Journal of Clinical Nutrition, (84), 475-482.

Yuhasz, M.S. (1974). Physical fitness Manual. Ontario, Canada: Ed. University of Western. 